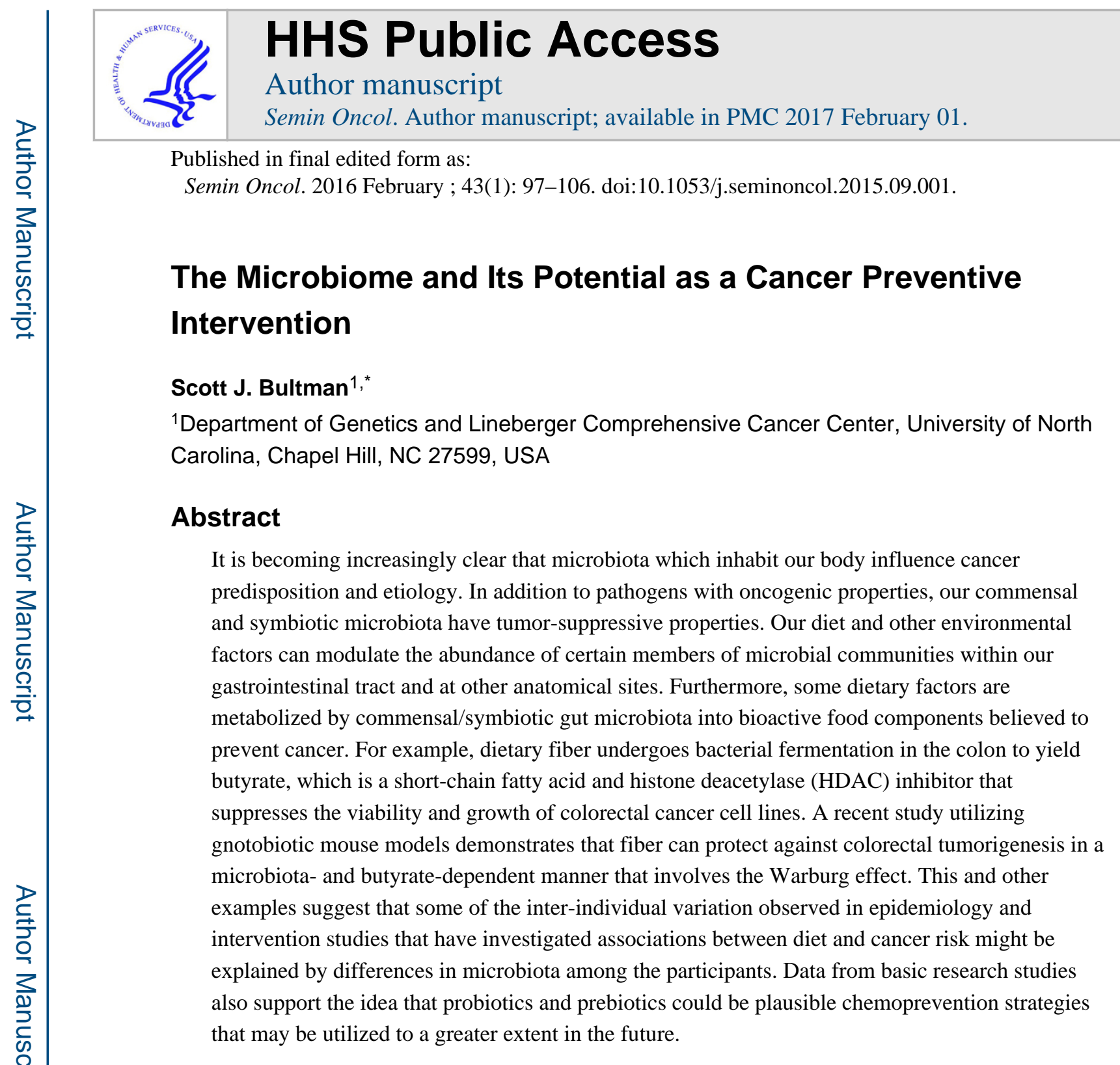

\title{
Introduction
}

Cancer is a leading cause of death that is associated with tremendous social and economic burdens. According to the National Cancer Institute (NCI), healthcare costs associated with the diagnosis and treatment of cancer in the United States currently exceed $\$ 125$ billion per year (1). This figure is projected to rise because of healthcare inflation and demographicsthe obesity epidemic and the aging of the United States population will undoubtedly increase the number of cases. Although targeted therapies such as imatinib (Gleevac) and trastuzumab (Herceptin) are efficacious at treating certain cancer subtypes, the vast majority

\footnotetext{
*To whom corresponding should be addressed: University of North Carolina, Department of Genetics, 120 Mason Farm Rd, Genetic Medicine Building, Room 5060. Chapel Hill, NC 27599-7264, USA. Tel: +1 919966 3359, Fax: +1 919843 4683,

Scott_Bultman@med.unc.edu.

Publisher's Disclaimer: This is a PDF file of an unedited manuscript that has been accepted for publication. As a service to our customers we are providing this early version of the manuscript. The manuscript will undergo copyediting, typesetting, and review of the resulting proof before it is published in its final citable form. Please note that during the production process errors may be discovered which could affect the content, and all legal disclaimers that apply to the journal pertain.
} 
of cancer cases still rely on conventional anticancer chemotherapeutics with varying degrees of efficacy and adverse effects. Therefore, a major goal is cancer prevention. It is estimated that $25-30 \%$ of cancer cases are due to tobacco use, $15-20 \%$ are due to infections, and 30 $35 \%$ of cancer cases are preventable via a healthy diet, physical activity, and maintaining a healthy body weight $(2,3)$. There is much interest in understanding the underlying mechanisms of cancer preventive effects, and it is becoming increasingly clear that the commensal microbiota that inhabit our body can inhibit pathogens from mounting infections and that they can also metabolize whole foods into bioactive food components that promote intestinal homeostasis and may prevent cancer. This review will discuss how microbiota that inhabit our body are detected and quantified followed by a discussion of cancer-prevention mechanisms and the prospect for probiotic and prebiotic strategies of cancer prevention.

\section{The human microbiome}

The human body harbors $\geq 10^{14}$ microbial cells, which is estimated to be $\sim 10$-fold greater than all of our somatic and germ cells combined (4). They are comprised of Bacteria, Archaea, Eukaryotes (such as yeast and other fungi), and viruses (including bacteriophage). Our microbiota and their collective genomes, which are referred to as the microbiome and harbor $~ 100$-fold more genes than the human genome, are being characterized by metagenomics approaches that combine next-generation sequencing with the computational analysis of targeted (16S rRNA hypervariable regions) and random (whole-genome shotgun) DNA sequence reads (5-7) (Figure 1). Based on these studies, we know that the composition of microbial communities varies across different anatomical sites $(8,9)$. Furthermore, these communities are dynamic rather than static because the composition of microbiota at any given site within an individual can change in response to diet and other lifestyle or environmental factors (10-12). We also know that the vast majority of these microbes are bacteria that reside within the lumen of our gastrointestinal (GI) tract. The commensal and symbiotic bacteria that live within our gut are protected from predators such as nematode roundworms, and they also benefit from a consistent supply of nutrients provided by our carbohydrate-rich diets (Figure 2). In return, many of the symbiotic bacteria digest glycans into disaccharides and monosaccharides for energy utilization by the human host as well as microbiota (Figure 2). To carry out this function, the gut microbiome is highly enriched for genes involved in carbohydrate metabolism including $\geq 115$ families of glycoside hydrolases and $\geq 21$ families of polysaccharide lyases $(13,14)$. In contrast, the human genome has relatively few genes that encode carbohydrate-metabolizing enzymes, presumably because mammals (and their genomes) co-evolved with gut microbiota (and the gut microbiome). As a result of this symbiotic relationship, gut microbiota are believed to improve our ability to absorb nutrients and extract calories from our diets $(15,16)$. Gut microbiota also produce essential vitamins such as vitamins K and B12 (17). Consequently, germfree mice, which are maintained devoid of all microbiota in gnotobiotic facilities (Figure 3), must be provided a diet that is fortified with additional vitamins other than those obtained through diet alone. 


\section{The microbiome and cancer}

Metagenomic sequencing projects have compared the composition of microbial communities in human disease cases to controls (Figure 1), and these association studies have implicated our microbiota in the prevention of many diseases including various types of cancer $(18,19)$. Normal diverse microbial communities can protect against cancer by multiple mechanisms. They can have an indirect effect by competing with pathogens for attachment sites, which limit pathogen abundance and prevent infections that drive carcinogenesis (Figure 4). The oncogenicity of certain pathogens such as human papillomavirus (HPV) and Helicobacter pylori is well established, but recent microbiome studies suggest commensals and opportunistic pathogens can also be involved and that infections associated with cancer might be more common than the current estimate of 15$20 \%$. For example, colorectal tumors are enriched for Fusobacterium nucleatum compared to normal colonic tissue (20-22). This bacterium was previously linked to periodontitis and appendicitis but not cancer. However, this association with cancer is not surprising because Fusobacterium nucleatum stimulates inflammation and can protect tumors from immune attack $(23,24)$. F. nucleatum also produces hydrogen sulfide in response to red meat consumption, which can induce DNA damage and genomic instability within in the colonic epithelium or developing tumors $(25,26)$. This increases colorectal cancer risk and progression, especially for individuals with mutations or perturbations in the DNA-damage response (e.g., ATR and ATM) in their germline or adenomas, respectively. Red meat consumption also increases cholesterol, which the liver uses to produce primary bile acids such as cholic acid that are conjugated to either glycine or taurine and undergo enterohepatic circulation. Approximately $5 \%$ of primary bile acids escape the enterohepatic circulation and reach the colon where specific bacteria deconjugate them (via bile salt hydrolases) and convert them into secondary bile acids (via dehydrogenation or dehydroxylation). At least one of these secondary bile acids, deoxycholic acid, causes DNA damage via the production of free radicals and has been implicated in liver, esophageal, and colorectal cancers $(27,28)$. These are prime examples of how our diet and microbiota can conspire to increase cancer risk.

The mucosa of colorectal cancer cases is enriched for Escherichia coli harboring a pks (polyketide synthase) pathogenicity island, which consists of a cluster of genes encoding enzymes that produce a genotoxic protein, collibactin, that can induce DNA damage in the host colonic epithelium (29-31). To demonstrate the importance of this pathogenicity island, Il10 $10^{-/}$mice were monoassociated with isogenic E. coli strains either containing or lacking (via a targeted deletion) pks in a gnotobiotic facility, and +pks increased the AOM (azoxymethane, a pro-carcinogen)-induced colorectal tumor burden without exacerbating inflammation (29). The mucosa of colorectal cancer cases is also enriched for enterotoxigenic Bacteroides fragilis, which corresponds to a subset of $\mathrm{B}$. fragilis strains that contain a pathogenicity island encoding a metalloproteinase (32-36). The bacterial-encoded metalloproteinase is believed to compromise the barrier function of the colonic epithelium, which exposes immune cells in the underlying lamina propria to luminal bacteria and bacterial gene products including lipopolysaccharide (LPS) and flagellin (Figure 4). This breach and exposure, in turn, leads to immune cell activation and inflammation, which is an 
emerging hallmark of cancer in general and colorectal cancer in particular $(37,38)$. It is likely that the B. fragilis toxin has additional oncogenic functions. It can cleave E-cadherin and activate the Wnt/ $\beta$-catenin pathway, and human-encoded metalloproteinases play an important role in tumor invasion and metastasis. Future studies will likely link additional microbiota to cancer, especially in cases where any one microbe has a subtle or modest effect while the combined effect of multiple microbes in aggregate is more robust. Microbes that have modest contributions may be dependent on genetic background of the host, which would result in greater inter-individual variation, and will be more difficult to detect than dominant pathogens capable of driving "one microbe-one disease" neoplasms such as HPV for cervical cancer and $\mathrm{H}$. pylori for gastric cancer. It is also possible that microbiome studies often overlook microbes that participate in early stages of tumorigenesis that are not involved in the later stages, especially if the growth or survival of these microbiota are selected against by the later-stage tumor microenvironment (e.g., low $\mathrm{pH}$ due to lactic acid because of the Warburg effect). Diet is known to influence the composition of our gut microbiota (10-12). Therefore, from a chemoprevention standpoint, a well-balanced diet can help maintain a "normal", albeit undefined, microbiome associated with good health and prevent an imbalance of a microbial community associated with diminished diversity, pathogenic infections, and increased cancer risk (referred to as dysbiosis).

Our commensal and symbiotic bacteria also diminish cancer risk in more direct ways than by inhibiting pathogens. This more direct route primarily involves their ability to metabolize dietary factors into bioactive food components, which can have cell-autonomous effects on the tumor or cell-of-origin as well as non-cell-autonomous effects targeting immune cells and other stromal cells in the tumor microenvironment (Figure 4). Our gut microbiota can be thought of as a second liver because of their prodigious metabolic capacity, which is not limited to calorie extraction, and the identification of microbial-derived metabolites that participate in disease prevention is a very active area of research (39). For this reason, the current trend is to move beyond basic microbiome studies, as outlined in Figure 1, and evaluate the effect of diet and other environmental factors on microbial abundance (metagenomics) plus microbial gene expression (metatranscriptomics and metaproteomics) and metabolite production (metabolomics). The next three sections focus on dietary fiber and colorectal cancer as an example of how gut microbiota can process bioactive food components into a metabolite relevant to chemoprevention.

\section{Dietary fiber and colorectal cancer prevention}

One of the most extensively studied dietary factors in chemoprevention has been fiber, which is defined as "the edible part of plants or their extracts, or analogous carbohydrates, that are resistant to digestion and absorption in the small intestine, but are utilized after partial or complete fermentation in the large intestine by resident microbiota" (40). Fiber includes polysaccharides (e.g., resistant starch, cellulose, hemicellulose, pectins, and gums), oligosaccharides, and lignins. As human populations have shifted away from traditional, high-fiber diets towards processed foods containing refined sugars, colorectal cancer incidence has increased markedly. Colorectal cancer is now the third most diagnosed cancer in both men and women in the United States, and it is also the third most deadly (41). This trend of increasing colorectal cancer incidence is most evident in China and developing 
countries that have rapidly adopted western diets in recent years (42). The correlation between decreased fiber consumption and increased colorectal incidence is also pronounced in developing countries because colonoscopies are performed on a limited basis. In contrast, widespread screening and removal of pre-cancerous adenomas in the United States has coincided with a recent decline in colorectal cancer incidence.

Yet the link between fiber consumption and prevention of colorectal adenomas and adenocarcinomas has been inconclusive, partly because ecologic studies, as described above and which provided the basis for Burkitt's original proposal that fiber is protective (43), are not rigorous. More rigorous prospective-cohort studies have been performed but have given rise to conflicting results (44-49), which has made this a controversial topic. However, it should be noted that these epidemiologic studies and mouse models of colorectal cancer have not controlled the composition of gut microbiota, which varies between individuals and is known to ferment fiber into short-chain fatty acids such as butyrate. Butyrate is highly abundant (present at $\mathrm{mM}$ levels in the lumen of the colon) and noteworthy because it has energetic and epigenetic functions in colonocytes and tumor-suppressive properties in colorectal cancer cell lines (50).

\section{Dietary fiber-microbiota-butyrate axis}

Recent studies have demonstrated that fiber consumption alters the composition of our gut microbiome to a greater extent than other dietary factors and increases the number of butyrate-producing bacteria (10-12). Furthermore, 25 microbiome studies have reported a significant decrease in butyrate-producing bacteria in human colorectal cancer cases compared to controls. However, one limitation of microbiome studies is that it is difficult to know whether a particular microbiome change is a cause or a consequence of the disease. For this reason, it is important to study mouse models maintained in gnotobiotic facilities where the microbiota can be manipulated (Figure 3). In addition to maintaining these mice in a germfree state, they can be colonized with one or more defined bacteria, which allows bacterial function in mammalian health and disease to be interrogated, as exemplified by the E. coli pks pathogenicity island study described above. To investigate dietary fiber and butyrate in a highly controlled manner, a mouse model of colorectal cancer was polyassociated with several bacteria in a gnotobiotic facility and provided control or highfiber diets that were otherwise essentially identical and isocaloric $(51,52)$. The high-fiber diet was provided from weaning (i.e., prior to tumor initiation) until the time of sacrifice and had a protective effect in mice colonized with a wild-type butyrate-producing bacterium but not in mice lacking a butyrate producer (Figure 5). The same mice colonized with a mutant strain of the butyrate-producing bacterium, which harbors a small deletion in the butyrate synthesis operon and produces diminished levels of butyrate, had an attenuated protective effect with an intermediate tumor burden (Figure 5). Furthermore, mice that completely lacked butyrate-producing bacteria but were provided a diet fortified with butyrate had a lower tumor burden than any of the other treatment groups(Figure 5). This is arguably the most convincing evidence that butyrate is a causal factor because it demonstrates that the fiber-microbiota chemopreventive effect can be recapitulated by exogenous butyrate. 
This study also explored the molecular mechanism of how butyrate functions as a microbialderived tumor-suppressive metabolite (51). Unlike most cell types in the body, which utilize glucose as their primary energy source, normal colonocytes rely on butyrate for $\sim 60-70 \%$ of their energy (53-55) (Figure 6). As a fatty acid, butyrate undergoes $\beta$-oxidation in the mitochondria, and this supports energy homeostasis necessary for the rapid cell proliferation of the colonic epithelium, which is renewed every $\sim 7$ days (it and the small intestinal epithelium arguably turn over faster than any other tissue in the body). In contrast, colorectal tumor cells (and tumor cells in general) undergo the Warburg effect and switch to glucose utilization and aerobic glycolysis (56) (Figure 7). As a result of this metabolic shift, butyrate is not metabolized in the mitochondria of tumor cells to the same extent and accumulates in the nucleus where it functions as a histone deacetylase (HDAC) inhibitor to epigenetically regulate gene expression $(51,57$ ) (Figure 6). In support of this model, butyrate (detected by LC-MS) and global histone acetylation levels (detected by immunohistochemistry and western blots) were elevated in tumors from mice that were colonized with the wild-type butyrate producer and provided a high-fiber diet, and this correlated with a lower tumor burden (Figure 5). Butyrate is a well-established $\operatorname{HDAC}$ inhibitor $(58,59)$, and butyrate target genes in tumors from mice provided a high-fiber diet included Fas and p21, which promote apoptosis and inhibit cell-cycle progression, respectively. This finding is compatible with the diminished tumor burden in these mice and the idea that butyrate is a tumor-suppressive metabolite.

However, the chemoprotective mechanism(s) could be more complicated than described above. Insoluble fibers such as cellulose are not fermented by gut microbiota and speed colonic transit. Decreased transit time is believed to be chemoprotective because it diminishes the exposure of colonocytes to ingested carcinogens such as heterocyclic amines from charred meats. Soluble fibers are fermented into short-chain fatty acids other than butyrate, such as acetate and propionate, and these or other metabolites could also contribute to chemoprevention. Finally, butyrate is a pleiotropic molecule that may function by additional mechanisms. In addition to functioning as an HDAC inhibitor, it can signal through certain $\mathrm{G}$ protein coupled receptors $(60,61)$. Butyrate could diminish tumorigenesis by attenuating inflammation. Butyrate enemas strongly ameliorate colonic inflammation associated with inflammatory bowel diseases (IBD) such as colitis and Crohn's disease in both rodent models and human patients (59). This is noteworthy because colitis patients have up to a 10 -fold increased risk of colorectal cancer $(38,62)$, which is consistent with the link between inflammation and cancer (37). Several recent studies demonstrate that butyrate activates FoxP3 expression in $\mathrm{CD}^{+}{ }^{+} \mathrm{T}$ cells and dendritic cells to induce the differentiation and expansion of immunosuppressive regulatory T (Treg) cells (63-66). Another recent study demonstrates that butyrate downregulates the production of pro-inflammatory cytokines by intestinal macrophages (67). It will need to be investigated whether these antiinflammatory events contribute to fiber-mediated chemoprevention.

\section{Translational potential of the dietary fiber-microbiota-butyrate axis}

The idea that butyrate is a tumor-suppressive metabolite is consistent with many published studies which have observed that butyrate inhibits the proliferation of colorectal cancer cell lines while stimulating their apoptosis and/or differentiation $(59,68)$. The gnotobiotic mouse 
experiments described above are valuable because they move beyond "factor dump" experiments where relatively high doses of butyrate are added to colorectal cancer cell lines in vitro. They demonstrate that dietary fiber and gut microbiota can modulate butyrate levels in the colonic lumen, and that this, in turn, can inhibit colorectal tumorigenesis in vivo where the colonic crypt architecture is intact and functions in the presence of stromal cells. These findings suggest that probiotics (butyrate-producing bacteria in this particular case) and/or prebiotics (soluble or "fermentable" fiber in this particular case) can be used to increase the levels of an endogenous HDAC inhibitor and diminish tumororigenesis. Unlike synthetic HDAC inhibitors, which are delivered systemically as chemotherapeutic agents for certain cancers, the probiotic/prebiotic approach should not have adverse effects for a couple of reasons (Figure 8). First, the bioavailability of butyrate is primarily restricted to the colon, which minimizes the chance of collateral damage in other tissues. Second, because butyrate is a naturally occurring fatty acid, it targets tumors cells in the colonic crypt. It is readily metabolized by normal colonocytes, whereas it accumulates as an HDAC inhibitor in tumor cells due to the Warburg effect.

It should be noted that the gnotobiotic mouse experimental design was reductionist in order to demonstrate that butyrate is a causal factor in chemoprevention. The mice were polyassociated with only several species of bacteria, which does not accurately model the complex microbiota that exist within the human GI tract. The semi-purified diets provided to the mice are not representative of our more varied diets that include different sources of fiber as well as higher levels of sugar, fat, and red meat, which may exacerbate cancer risk and could possibly mask a beneficial fiber effect. Additionally, the mice were provided a high-fiber diet at weaning prior to tumor initiation (via azoxymethane [AOM] injection), and it is not clear whether fiber would still be protective if provided after the onset of tumorigenesis. Nevertheless, despite these caveats, human adenocarcinomas were shown to have higher levels of butyrate and histone acetylation than normal colonic samples (51). This finding suggests that the gnotobiotic mouse data may be relevant to human cancer prevention. Based on this knowledge, it would be interesting to revisit prospective-cohort studies that have investigated whether there is a link between fiber consumption and colorectal cancer but combine them with microbiome studies. The hypothesis would be that if microbiome differences among the participants were taken into account, then it would be possible to discriminate between those individuals who respond to the putative chemoprotective effect of fiber and non-responders. This would resolve some of the conflicting results from previous human studies and possibly confirm butyrate as an important molecule in human chemoprevention.

\section{Other bacterial metabolites and cancers}

Although the vast majority of our microbiota reside in our gut, they can influence diseases beyond our GI tract, such as cardiovascular disease and autism, and this applies to cancer. Many gut microbe-derived metabolites have a much broader bioavailability than butyrate, and the following paragraphs in this section provide some examples relevant to cancer prevention. It should also be noted that bacterial densities are relatively high in close proximity to the mucous membranes of other tissues such as the lung and urogenital tract epithelia. This physical relationship suggests that local microbial communities will also 
influence the initiation and progression of carcinomas at these sites. It is also possible that microbiota will influence leukemias/lymphomas to a greater extent than other cancers because our hematopoietic lineages play a key role in the inflammatory response to microbes and microbial products.

Dietary polyphenols, which include flavonoids (e.g., quercetin and kaempferol), phenolic acids, anthrocyanins, and lignins present in tea, wine, fruits, nuts, and vegetables, have received extensive attention because of their chemoprotective effects in mouse models and human epidemiology studies. Resveratrol has probably received the most attention because it is a caloric-restriction mimetic that is pleiotropic and benefits health in multiple ways. However, it is not the best example for being metabolized by gut microbiota. Although gut microbiota can convert it to trans-resveratrol metabolites such as dihydroresveratrol, there are considerable inter-individual differences and the functional relevance of these derivatives is poorly understood (69). In contrast, ellagic acid is a polyphenol present in certain berries and nuts that is an anti-oxidant with cancer-preventive properties. Ellagic acid is metabolized by colonic microbiota into urolithins that have pro-estrogenic and antiestrogenic activities in a context-dependent manner (70) (Table 1). Urolithins can also downregulate COX-2 to lower prostaglandin production and inflammation so the anti-cancer effects apparently involve multiple pathways (71). Another polyphenol is daidzein, which is a soy-based isoflavone metabolized by gut microbiota into equol (72) (Table 1). Equol can be detected in only $30-40 \%$ of individuals, and, although the reason for this is not understood, it could be due, in part, to the relative abundance of specific bacteria $(73,74)$. The ability to produce equol is positively correlated with sulfate-reducing bacteria and negatively correlated with Clostridium coccoides and Eubacterium rectale (75). Some epidemiologic studies have reported correlations between equol or equol-producing bacteria and diminished breast cancer risk in women and diminished prostate cancer risk in men. However, these correlations have been observed in Asian populations but not European populations (76). It is not clear whether these ethnic disparities are due to differences in genetics, microbiota, or diet (e.g., soy consumption), and more work will be required to strengthen the link between equol and cancer prevention. Generally speaking, based on situations like this, it would be advantageous to combine epidemiology studies with GWAS (genome-wide association studies) or exome sequencing as well as microbiome studies. This kind of integrated approach might allow a combination of factors to be identified that have a significant and reproducible effect regarding diet and chemoprevention. In addition, it would be cost effective and useful to assess relatively short-term probiotic and prebiotic regimens by performing metabolomics or analyzing cancer-related biomarkers as surrogates and then use this information to direct more expensive, longer-term epidemiology studies or intervention trials.

Cruciferous vegetables such as broccoli and cabbage contain high levels of glucosinolates. When these vegetables are uncooked and either chopped or chewed, plant-derived myrosinases convert the glucosinolates to isothiocyanates (ITC) such as sulforaphane (which is an HDAC inhibitor like butyrate) that have anti-carcinogenic properties in cell lines and mouse models and might diminish human cancer risk (particularly for lung, breast, prostate, colorectal, and prostate although the epidemiology results are mixed). However, cruciferous vegetables are usually cooked. Although this denatures the plant-derived 
myrosinases, bacteria-derived thioglucosidases can convert glucosinolates into ITC in the gut to exert their beneficial effects (74) (Table 1).

Linoleic acid is an omega-6 polyunsaturated fatty acid (PUFA) that is a constituent of vegetable oils. Because linoleic acid is a precursor of arachadonic acid, which gives rise to prostaglandins and inflammation, high intake of vegetable oils can alter the omega- 6 to omega-3 ratio and be deleterious. However, certain gut microbiota, including strains of Lactobacilli and Bifidobacteria used in probiotics, can conjugate linoleic acid (73). Not only does this bacterial conjugation diminish linoleic acid levels, but some conjugated linoleic acid isomers are reported to have anti-inflammatory and anti-carcinogenic properties (Table $1)$.

\section{Probiotics, prebiotics, and the growing functional food/nutraceutical industry}

The previous section provided some examples of how our gut microbiota influence dietary components to potentially prevent cancer (summarized in Table 1), but the reverse is also true. In fact, many more food components are known to influence the composition of our gut microbiota. Undoubtedly, many more will be discovered and some diet-induced changes in microbiota are likely to benefit human health in various ways, including preventing cancer. Of course, there are the prebiotics, which have been referred to in above sections and are defined as indigestible food ingredients that selectively stimulate the growth and/or activity of certain gut microbiota that confer a health benefit. A number of prebiotics have been implicated in cancer prevention including various sources of dietary fiber such as inulin that promote the growth of Bifidobacteria (73).

A more direct microbial intervention involves probiotics, which have also been referred to in previous sections and correspond to live microorganisms present in foods or dietary supplements that confer a health benefit. Although there is not much evidence for cancer prevention, probiotic bacteria have been implicated in a number of other health outcomes. Lactobacilli in yogurt is arguably the best-known example, but Streptococci and Bifidobacteria in cheeses and other foods and drinks are also common. One benefit of Lactobacilli in yogurt is improved digestion of dairy products in individuals who are lactose intolerant. This allows some people to increase their intake of calcium and is noteworthy because the prevalence of lactose intolerance ranges from 5-15\% in northern European countries and the United States to $>50 \%$ in African and Asian countries (77). The beneficial effect is due to live bacteria, which provide $\beta$ galactosidase (lactase) activity, because heated or pasteurized yogurt is not effective (77). Microbiome studies suggest that yogurt must be consumed on a regular basis (probably daily) to maintain elevated levels of Lactobacilli (12). Probiotics can be improved by supplementing foods with bacteria engineered either to have stronger beneficial effects or to more stably colonize the human GI tract. For example, although Lactobacillus acidophilus is a beneficial bacteria commonly utilized as a probiotic, a glycolipid present in the cell wall, lipoteichoic acid, can potentially have adverse effects by stimulating inflammation via Toll-like receptor 2 and cytokine production $(78,79)$. To address this concern, a strain of Lactobacillus acidophilus was engineered with a deletion in the phosphoglycerol transferase gene that is unable to synthesize lipoteichoic acid, and oral 
administration of this bacterium to $\mathrm{Apc}^{\Delta \text { floxed mice resulted in the regression of already }}$ established colonic polyps (80). Strains of Lactobacillus casei and Lactococcus lactis have also been engineered to produce a protein called elafin that diminished inflammation in a mouse model of colitis (81). When these bacteria were added to inflamed epithelial cells from human colitis patients ex vivo, they attenuated cytokine production and cell permeability. A final example is a strain of Lactobacillus gasseri engineered to overexpress the antioxidant superoxide dismutase that decreased colitis in IL-10 knockout mice (82). Delivery of these Lactobacilli as improved probiotics would be considered functional foods or nutraceuticals, which usually do not involve microbes and include vitamin-fortified foods such as golden rice (a genetically-modified crop engineered to contain beta carotene) and various foods fortified with omega-3 PUFAs (e.g., cereal, pork, eggs). The functional food/ nutraceutical market is growing rapidly; in the United States, it has increased by $31 \%$ since 2006 to a value of $\$ 7.5$ billion (and $\$ 24$ billion globally). Although functional foods and nutraceuticals will likely prove useful for many individuals, many unsubstantiated claims are made by this industry so products must be evaluated on a case-by-case basis. This is particularly true for dietary supplements because they do not require Food and Drug Administration (FDA) approval.

Probiotic and prebiotic strategies will prove more efficacious than antibiotic treatments for the purpose of chemoprevention. Although antibiotics have been shown to decrease the tumor burden of some mouse models (most likely via diminished inflammation), they are not good candidates for chemoprevention or chemotherapeutic adjuvants in the clinic. Not only does the overuse of antibiotics make the problem of antibiotic-resistant bacterial strains even worse, but it kills many commensal/symbiotic bacteria including some that likely promote homeostasis and protect against carcinogenesis. Furthermore, not all bacteria return to normal levels following antibiotic treatment (12). It has been proposed that our pervasive antibiotics usage is altering our microbiota and contributing to the increased incidence of obesity, IBD, allergies, and asthma (83), and this might also apply to certain cancers. In fact, as $\mathrm{H}$. pylori has been erradicated to a large extent, gastric cancer has decreased but esophageal cancer has became more common. One possibility is that $\mathrm{H}$. pylori can alter stomach $\mathrm{pH}$ and acid reflux in a manner that protects against Barrett's esophagus and esophageal cancer (83). Therefore, instead of using antibiotics to kill bacteria indiscriminately, it would be better to take steps that maintain or restore a beneficial microbial composition. This is the basis for fecal microbiota transplantations (which can be considered a probiotic treatment) that are very effective for the treatment of diarrhea in people with severe Clostridium difficile infections, which usually arise because antibiotics eliminated commensal bacteria that are capable of displacing or suppressing C. difficile. Although the notion that global transplantation of microbiota might function as a cancer preventive intervention is not yet a reality, the application of such bacteriotherapy to the prevention setting holds promise for the future.

\section{Future directions}

We have not yet developed culture conditions that support the growth of most microbes that inhabit the human body, particularly anaerobic bacteria that reside deep within our GI tract. This limitation has not prevented us from using metagenomics to characterize microbial 
populations and to identify microbiome differences between individuals with certain diseases including cancer compared to controls. It is important that microbiome studies continue and that they become integrated with epidemiology studies (especially with respect to diet), GWAS, and metabolomics. However, it will become increasingly important that we are able to culture specific bacteria so they can be analyzed in gnotobiotic mouse models. This approach will allow us to move from correlation to causation and will provide insight into molecular mechanisms, which may lead to improved probiotic/prebiotic strategies of disease prevention.

\section{References}

1. Mariotto AB, Yabroff KR, Shao Y, Feuer EJ, Brown ML. Projections of the cost of cancer care in the United States: 2010-2020. Journal of the National Cancer Institute. 2011; 103(2):117-128. [PubMed: 21228314]

2. Anand $\mathrm{P}$, et al. Cancer is a preventable disease that requires major lifestyle changes. Pharmaceutical research. 2008; 25(9):2097-2116. [PubMed: 18626751]

3. Willett WC. Diet and cancer. The oncologist. 2000; 5(5):393-404. [PubMed: 11040276]

4. Savage DC. Microbial ecology of the gastrointestinal tract. Annual review of microbiology. 1977; 31:107-133.

5. Anonymous. A framework for human microbiome research. Nature. 2012; 486(7402):215-221. [PubMed: 22699610]

6. Goodrich JK, et al. Conducting a microbiome study. Cell. 2014; 158(2):250-262. [PubMed: 25036628]

7. Weinstock GM. Genomic approaches to studying the human microbiota. Nature. 2012; 489(7415): 250-256. [PubMed: 22972298]

8. Anonymous. Structure, function and diversity of the healthy human microbiome. Nature. 2012; 486(7402):207-214. [PubMed: 22699609]

9. Costello EK, et al. Bacterial community variation in human body habitats across space and time. Science. 2009; 326(5960):1694-1697. [PubMed: 19892944]

10. Turnbaugh PJ, et al. The effect of diet on the human gut microbiome: a metagenomic analysis in humanized gnotobiotic mice. Science translational medicine. 2009; 1(6):6ra14.

11. David LA, et al. Diet rapidly and reproducibly alters the human gut microbiome. Nature. 2014; 505(7484):559-563. [PubMed: 24336217]

12. David LA, et al. Host lifestyle affects human microbiota on daily timescales. Genome biology. 2014; 15(7):R89. [PubMed: 25146375]

13. Gill SR, et al. Metagenomic analysis of the human distal gut microbiome. Science. 2006; 312(5778):1355-1359. [PubMed: 16741115]

14. Ley RE, Turnbaugh PJ, Klein S, Gordon JI. Microbial ecology: human gut microbes associated with obesity. Nature. 2006; 444(7122):1022-1023. [PubMed: 17183309]

15. Dethlefsen L, McFall-Ngai M, Relman DA. An ecological and evolutionary perspective on humanmicrobe mutualism and disease. Nature. 2007; 449(7164):811-818. [PubMed: 17943117]

16. Ley RE, et al. Evolution of mammals and their gut microbes. Science. 2008; 320(5883):16471651. [PubMed: 18497261]

17. Wostmann BS. The germfree animal in nutritional studies. Annual review of nutrition. 1981; $1: 257-279$.

18. Bultman SJ. Emerging roles of the microbiome in cancer. Carcinogenesis. 2014; 35(2):249-255. [PubMed: 24302613]

19. Schwabe RF, Jobin C. The microbiome and cancer. Nature reviews. Cancer. 2013; 13(11):800812. [PubMed: 24132111]

20. Castellarin M, et al. Fusobacterium nucleatum infection is prevalent in human colorectal carcinoma. Genome research. 2012; 22(2):299-306. [PubMed: 22009989] 
21. Kostic AD, et al. Genomic analysis identifies association of Fusobacterium with colorectal carcinoma. Genome research. 2012; 22(2):292-298. [PubMed: 22009990]

22. McCoy AN, et al. Fusobacterium is associated with colorectal adenomas. PloS one. 2013; 8(1):e53653. [PubMed: 23335968]

23. Garrett WS. Cancer and the microbiota. Science. 2015; 348(6230):80-86. [PubMed: 25838377]

24. Gur C, et al. Binding of the Fap2 protein of Fusobacterium nucleatum to human inhibitory receptor TIGIT protects tumors from immune cell attack. Immunity. 2015; 42(2):344-355. [PubMed: 25680274]

25. Huycke MM, Gaskins HR. Commensal bacteria, redox stress, and colorectal cancer: mechanisms and models. Exp Biol Med (Maywood). 2004; 229(7):586-597. [PubMed: 15229352]

26. Rooks MG, Garrett WS. Bacteria, food, and cancer. F1000 biology reports. 2011; 3:12. [PubMed: 21876723]

27. Nagengast FM, Grubben MJ, van Munster IP. Role of bile acids in colorectal carcinogenesis. Eur J Cancer. 1995; 31A(7-8):1067-1070. [PubMed: 7576993]

28. Yoshimoto S, et al. Obesity-induced gut microbial metabolite promotes liver cancer through senescence secretome. Nature. 2013; 499(7456):97-101. [PubMed: 23803760]

29. Arthur JC, et al. Intestinal inflammation targets cancer-inducing activity of the microbiota. Science. 2012; 338(6103):120-123. [PubMed: 22903521]

30. Buc E, et al. High prevalence of mucosa-associated E. coli producing cyclomodulin and genotoxin in colon cancer. PloS one. 2013; 8(2):e56964. [PubMed: 23457644]

31. Cuevas-Ramos G, et al. Escherichia coli induces DNA damage in vivo and triggers genomic instability in mammalian cells. Proceedings of the National Academy of Sciences of the United States of America. 2010; 107(25):11537-11542. [PubMed: 20534522]

32. Goodwin AC, et al. Polyamine catabolism contributes to enterotoxigenic Bacteroides fragilisinduced colon tumorigenesis. Proceedings of the National Academy of Sciences of the United States of America. 2011; 108(37):15354-15359. [PubMed: 21876161]

33. Sears CL. Enterotoxigenic Bacteroides fragilis: a rogue among symbiotes. Clinical microbiology reviews. 2009; 22(2):349-369. Table of Contents. [PubMed: 19366918]

34. Shiryaev SA, et al. Structural and functional diversity of metalloproteinases encoded by the Bacteroides fragilis pathogenicity island. The FEBS journal. 2014; 281(11):2487-2502. [PubMed: 24698179]

35. Boleij A, et al. The Bacteroides fragilis Toxin Gene Is Prevalent in the Colon Mucosa of Colorectal Cancer Patients. Clinical infectious diseases : an official publication of the Infectious Diseases Society of America. 2014

36. Sears CL, Geis AL, Housseau F. Bacteroides fragilis subverts mucosal biology: from symbiont to colon carcinogenesis. The Journal of clinical investigation. 2014; 124(10):4166-4172. [PubMed: 25105360]

37. Hanahan D, Weinberg RA. Hallmarks of cancer: the next generation. Cell. 2011; 144(5):646-674. [PubMed: 21376230]

38. Terzic J, Grivennikov S, Karin E, Karin M. Inflammation and colon cancer. Gastroenterology. 2010; 138(6):2101-2114. e2105. [PubMed: 20420949]

39. Nicholson JK, et al. Host-gut microbiota metabolic interactions. Science. 2012; 336(6086):12621267. [PubMed: 22674330]

40. Lim CC, Ferguson LR, Tannock GW. Dietary fibres as "prebiotics": implications for colorectal cancer. Molecular nutrition \& food research. 2005; 49(6):609-619. [PubMed: 15864790]

41. Siegel R, Naishadham D, Jemal A. Cancer statistics, 2013. CA: a cancer journal for clinicians. 2013; 63(1):11-30. [PubMed: 23335087]

42. Center MM, Jemal A, Ward E. International trends in colorectal cancer incidence rates. Cancer epidemiology, biomarkers \& prevention : a publication of the American Association for Cancer Research, cosponsored by the American Society of Preventive Oncology. 2009; 18(6):1688-1694.

43. Burkitt DP, Walker AR, Painter NS. Effect of dietary fibre on stools and the transit-times, and its role in the causation of disease. Lancet. 1972; 2(7792):1408-1412. [PubMed: 4118696] 
44. Baron JA. Dietary fiber and colorectal cancer: an ongoing saga. JAMA : the journal of the American Medical Association. 2005; 294(22):2904-2906. [PubMed: 16352800]

45. Bingham SA, et al. Dietary fibre in food and protection against colorectal cancer in the European Prospective Investigation into Cancer and Nutrition (EPIC): an observational study. Lancet. 2003; 361(9368):1496-1501. [PubMed: 12737858]

46. Ferguson LR, Harris PJ. The dietary fibre debate: more food for thought. Lancet. 2003; 361(9368): 1487-1488. [PubMed: 12737854]

47. Fuchs CS, et al. Dietary fiber and the risk of colorectal cancer and adenoma in women. The New England journal of medicine. 1999; 340(3):169-176. [PubMed: 9895396]

48. Park Y, et al. Dietary fiber intake and risk of colorectal cancer: a pooled analysis of prospective cohort studies. JAMA : the journal of the American Medical Association. 2005; 294(22):28492857. [PubMed: 16352792]

49. Goodlad RA. Dietary fibre and the risk of colorectal cancer. Gut. 2001; 48(5):587-589. [PubMed: $11302948]$

50. Bultman SJ. Molecular pathways: gene-environment interactions regulating dietary fiber induction of proliferation and apoptosis via butyrate for cancer prevention. Clinical cancer research : an official journal of the American Association for Cancer Research. 2014; 20(4):799-803. [PubMed: 24270685]

51. Donohoe DR, et al. A Gnotobiotic Mouse Model Demonstrates That Dietary Fiber Protects against Colorectal Tumorigenesis in a Microbiota- and Butyrate-Dependent Manner. Cancer discovery. 2014; 4(12):1387-1397. [PubMed: 25266735]

52. Sebastian C, Mostoslavsky R. Untangling the fiber yarn: butyrate feeds warburg to suppress colorectal cancer. Cancer discovery. 2014; 4(12):1368-1370. [PubMed: 25477104]

53. Donohoe DR, et al. The microbiome and butyrate regulate energy metabolism and autophagy in the mammalian colon. Cell metabolism. 2011; 13(5):517-526. [PubMed: 21531334]

54. Roediger WE. Role of anaerobic bacteria in the metabolic welfare of the colonic mucosa in man. Gut. 1980; 21(9):793-798. [PubMed: 7429343]

55. Roediger WE. Utilization of nutrients by isolated epithelial cells of the rat colon. Gastroenterology. 1982; 83(2):424-429. [PubMed: 7084619]

56. Vander Heiden MG, Cantley LC, Thompson CB. Understanding the Warburg effect: the metabolic requirements of cell proliferation. Science. 2009; 324(5930):1029-1033. [PubMed: 19460998]

57. Donohoe DR, et al. The Warburg effect dictates the mechanism of butyrate-mediated histone acetylation and cell proliferation. Molecular cell. 2012; 48(4):612-626. [PubMed: 23063526]

58. Davie JR. Inhibition of histone deacetylase activity by butyrate. The Journal of nutrition. 2003; 133(7 Suppl):2485S-2493S. [PubMed: 12840228]

59. Hamer HM, et al. Review article: the role of butyrate on colonic function. Alimentary pharmacology \& therapeutics. 2008; 27(2):104-119. [PubMed: 17973645]

60. Goncalves P, Martel F. Butyrate and colorectal cancer: the role of butyrate transport. Current drug metabolism. 2013; 14(9):994-1008. [PubMed: 24160296]

61. Thangaraju M, et al. GPR109A is a G-protein-coupled receptor for the bacterial fermentation product butyrate and functions as a tumor suppressor in colon. Cancer research. 2009; 69(7):28262832. [PubMed: 19276343]

62. Itzkowitz SH, Harpaz N. Diagnosis and management of dysplasia in patients with inflammatory bowel diseases. Gastroenterology. 2004; 126(6):1634-1648. [PubMed: 15168373]

63. Arpaia N, et al. Metabolites produced by commensal bacteria promote peripheral regulatory T-cell generation. Nature. 2013; 504(7480):451-455. [PubMed: 24226773]

64. Furusawa Y, et al. Commensal microbe-derived butyrate induces the differentiation of colonic regulatory T cells. Nature. 2013; 504(7480):446-450. [PubMed: 24226770]

65. Singh N, et al. Activation of Gpr109a, receptor for niacin and the commensal metabolite butyrate, suppresses colonic inflammation and carcinogenesis. Immunity. 2014; 40(1):128-139. [PubMed: 24412617]

66. Smith PM, et al. The microbial metabolites, short-chain fatty acids, regulate colonic Treg cell homeostasis. Science. 2013; 341(6145):569-573. [PubMed: 23828891] 
67. Chang PV, Hao L, Offermanns S, Medzhitov R. The microbial metabolite butyrate regulates intestinal macrophage function via histone deacetylase inhibition. Proceedings of the National Academy of Sciences of the United States of America. 2014; 111(6):2247-2252. [PubMed: 24390544]

68. Fung KY, Cosgrove L, Lockett T, Head R, Topping DL. A review of the potential mechanisms for the lowering of colorectal oncogenesis by butyrate. The British journal of nutrition. 2012; 108(5): 820-831. [PubMed: 22676885]

69. Bode LM, et al. In vivo and in vitro metabolism of trans-resveratrol by human gut microbiota. The American journal of clinical nutrition. 2013; 97(2):295-309. [PubMed: 23283496]

70. Larrosa M, Gonzalez-Sarrias A, Garcia-Conesa MT, Tomas-Barberan FA, Espin JC. Urolithins, ellagic acid-derived metabolites produced by human colonic microflora, exhibit estrogenic and antiestrogenic activities. Journal of agricultural and food chemistry. 2006; 54(5):1611-1620. [PubMed: 16506809]

71. Gonzalez-Sarrias A, Larrosa M, Tomas-Barberan FA, Dolara P, Espin JC. NF-kappaB-dependent anti-inflammatory activity of urolithins, gut microbiota ellagic acid-derived metabolites, in human colonic fibroblasts. The British journal of nutrition. 2010; 104(4):503-512. [PubMed: 20338073]

72. Atkinson C, Frankenfeld CL, Lampe JW. Gut bacterial metabolism of the soy isoflavone daidzein: exploring the relevance to human health. Exp Biol Med (Maywood). 2005; 230(3):155-170. [PubMed: 15734719]

73. Davis CD, Milner JA. Gastrointestinal microflora, food components and colon cancer prevention. The Journal of nutritional biochemistry. 2009; 20(10):743-752. [PubMed: 19716282]

74. Hullar MA, Burnett-Hartman AN, Lampe JW. Gut microbes, diet, and cancer. Cancer treatment and research. 2014; 159:377-399. [PubMed: 24114492]

75. Bolca $S$, et al. Microbial and dietary factors are associated with the equol producer phenotype in healthy postmenopausal women. The Journal of nutrition. 2007; 137(10):2242-2246. [PubMed: 17885005]

76. Lampe JW. Emerging research on equol and cancer. The Journal of nutrition. 2010; 140(7):1369S1372S. [PubMed: 20505018]

77. Guarner F, Malagelada JR. Gut flora in health and disease. Lancet. 2003; 361(9356):512-519. [PubMed: 12583961]

78. Mayer ML, Phillips CM, Townsend RA, Halperin SA, Lee SF. Differential activation of dendritic cells by Toll-like receptor agonists isolated from the Gram-positive vaccine vector Streptococcus gordonii. Scandinavian journal of immunology. 2009; 69(4):351-356. [PubMed: 19284500]

79. Mayer ML, Phillips CM, Stadnyk AW, Halperin SA, Lee SF. Synergistic BM-DC activation and immune induction by the oral vaccine vector Streptococcus gordonii and exogenous tumor necrosis factor. Molecular immunology. 2009; 46(8-9):1883-1891. [PubMed: 19278729]

80. Khazaie K, et al. Abating colon cancer polyposis by Lactobacillus acidophilus deficient in lipoteichoic acid. Proceedings of the National Academy of Sciences of the United States of America. 2012; 109(26):10462-10467. [PubMed: 22689992]

81. Motta JP, et al. Food-grade bacteria expressing elafin protect against inflammation and restore colon homeostasis. Science translational medicine. 2012; 4(158):158ra144.

82. Carroll IM, et al. Anti-inflammatory properties of Lactobacillus gasseri expressing manganese superoxide dismutase using the interleukin 10-deficient mouse model of colitis. American journal of physiology. Gastrointestinal and liver physiology. 2007; 293(4):G729-G738. [PubMed: 17640978]

83. Blaser M. Antibiotic overuse: Stop the killing of beneficial bacteria. Nature. 2011; 476(7361):393394. [PubMed: 21866137] 

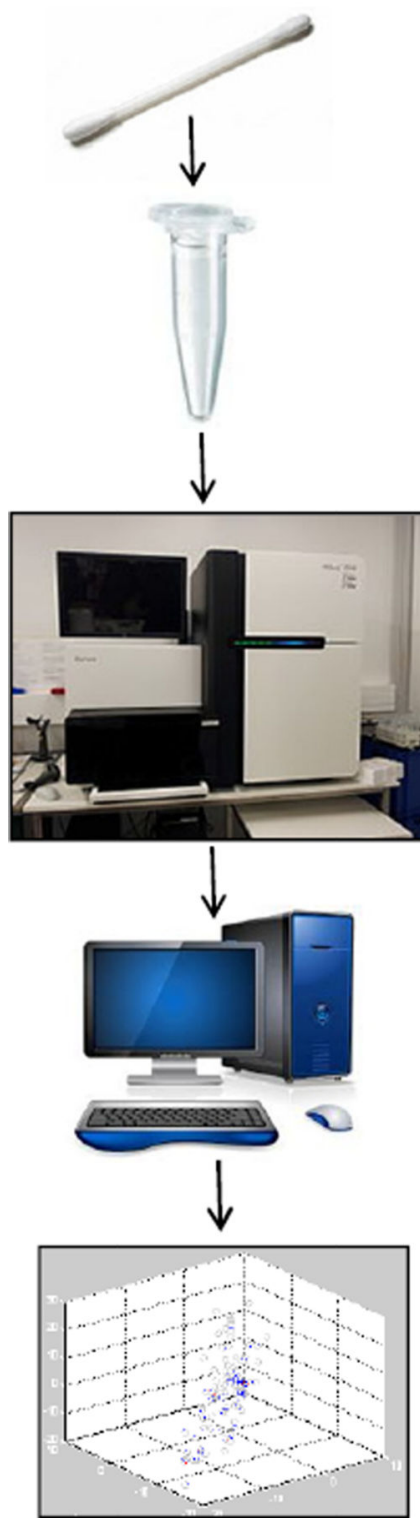

Biological material (buccal swabs, stool samples, tissue biopsies, etc.): - Can be obtained from disease cases and controls

Prepare genomic DNA

\section{Next-generation DNA sequencing: \\ -16 S rRNA \\ -whole-genome shotgun (discard human sequence reads)}

Computational assembly and analysis of microbial sequence reads to quantify abundance of microbiota

Figure 1.

A flowchart showing basic steps of a microbiome study. See cited references for details. 

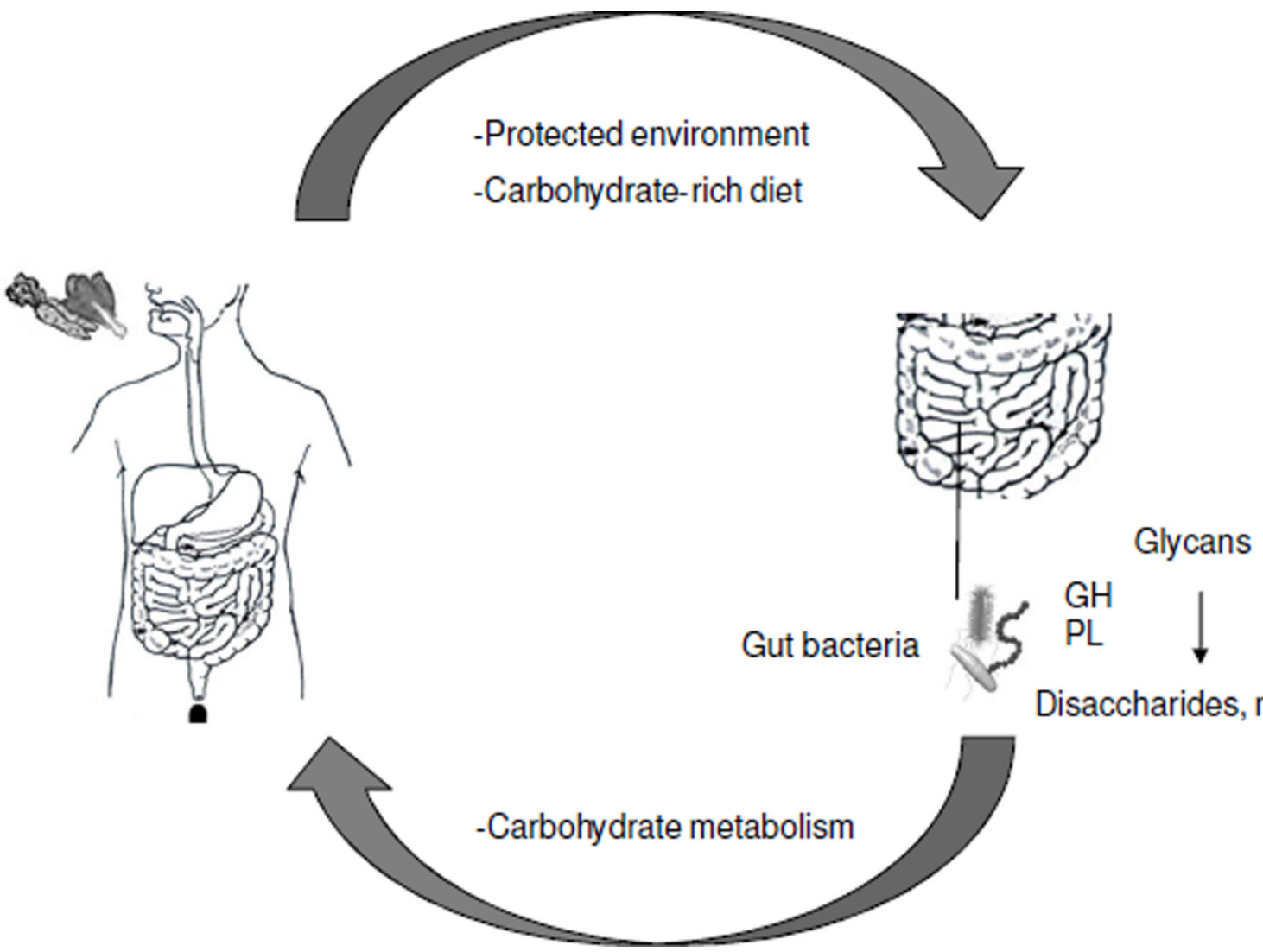

Disaccharides, monosaccharides

Figure 2.

A symbiotic relationship exists between humans and our gut microbiota regarding energy homeostasis. Humans provide gut bacteria with a protected environment and carbohydrates. The gut microbiome is enriched for genes that encode enzymes such as glycoside hydrolases $(\mathrm{GH})$ and polysaccharide lyases (PL) that catabolize complex carbohydrates into simpler sugars that are utilized by both the gut microbiota and us as their human hosts. 


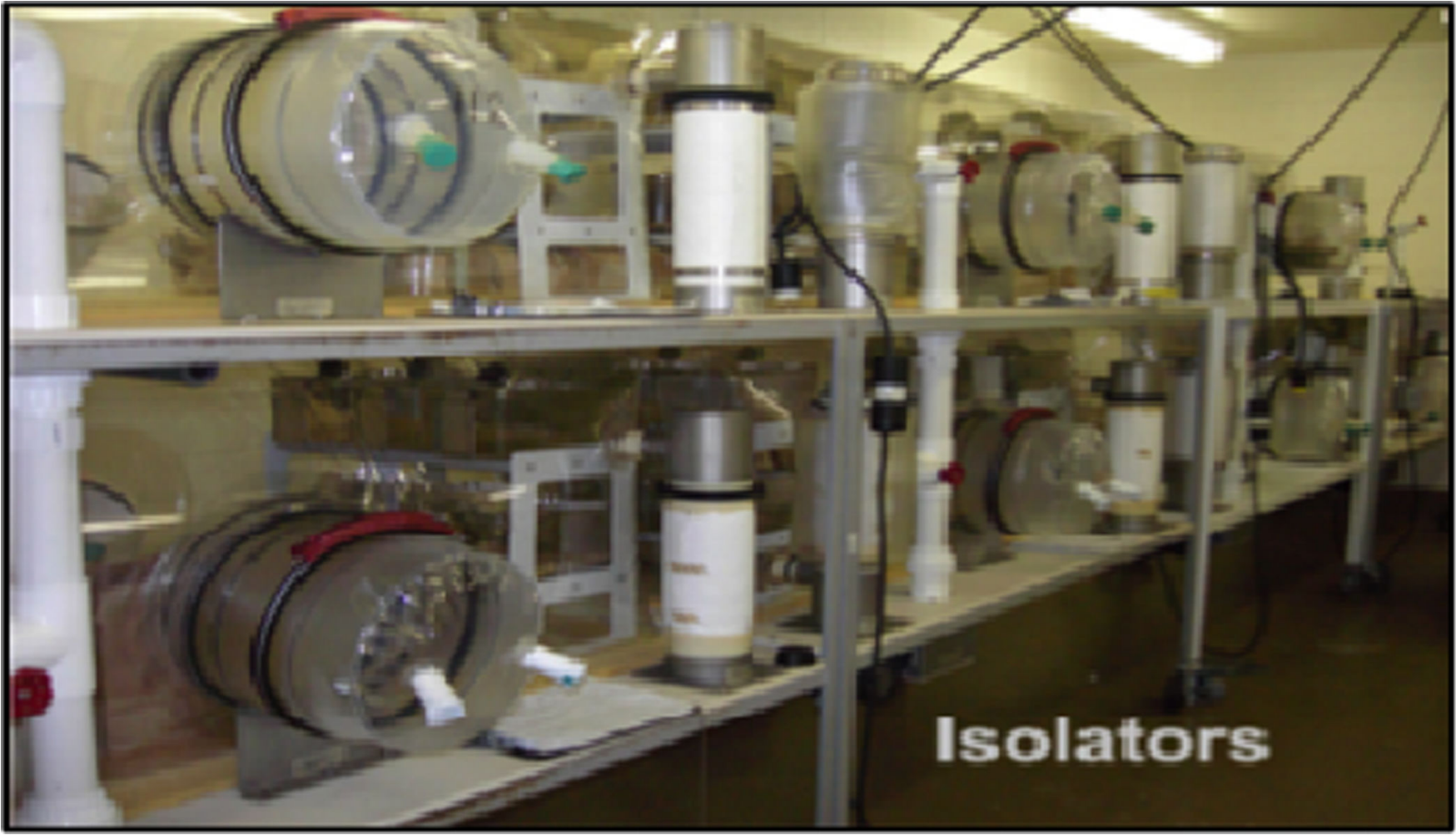

Figure 3.

Gnotobiotic mouse models of cancer are crucial for probing the importance of microbiota in chemoprevention. Image of a gnotobiotic mouse facility. Mice are maintained in isolators ( $\mathrm{n}=6$ are pictured) where the air is filtered with a specialized HEPA filtration system (white cylinders). Everything that enters the isolators (e.g., cages, bedding, food, water) is autoclaved in advance (usually inside of metal canisters) and enters through an airlock system (circular structures) after the exterior surface is sprayed with antiseptic. The mice are handled using a glovebox apparatus (not pictured, located on other side of isolators). The mice can be maintained germfree (i.e., devoid of all microbiota) or they can be colonized with one or more specific microbiota introduced by gavage. The mice can be "humanized" by colonizing them with human-derived microbiota, including from disease cases versus controls, to interrogate the function of human microbiota in a rigorously controlled manner. A typical isolator holds up to $\sim 12$ cages. To prevent contamination, only one combination of microbiota can be used per isolator and regular microbiology testing is necessary (e.g., fecal samples and swabs analyzed by Gram staining, PCR, etc.). 
Lumen

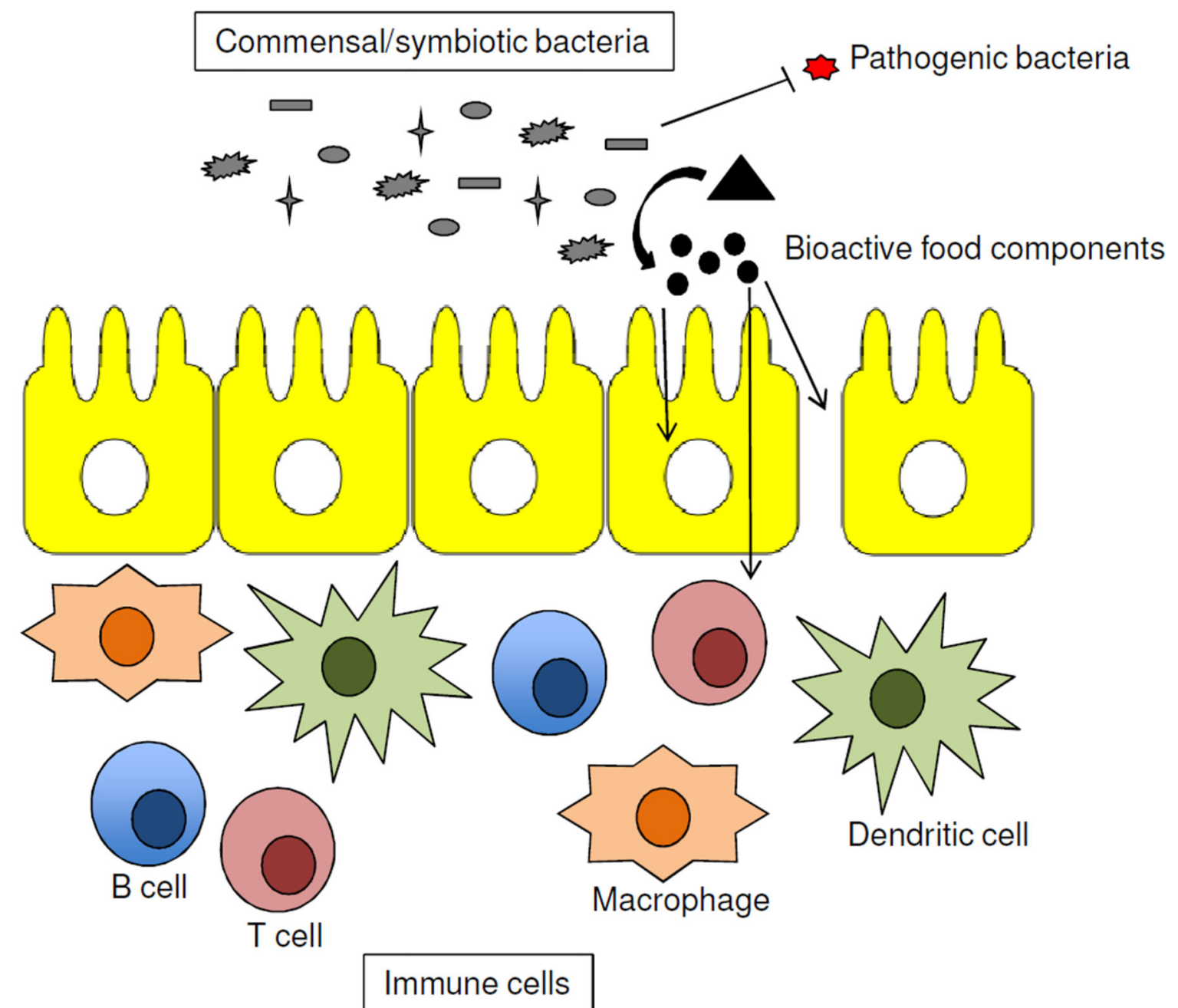

Intestinal Epithelium

Lamina

Propria

Figure 4.

Commensal/symbiotic microbiota protect against cancer by multiple mechanisms.

Schematic of intestine showing 3 compartments: top, the lumen containing microbiota (gray); middle, a single layer of epithelial cells; bottom, an underlying lamina propria containing immune cells. Commensal/symbiotic bacteria can prevent cancer indirectly by outcompeting cancer-causing pathogens and more directly by metabolizing dietary factors into bioactive food components (circles). The latter can have cell autonomous effects within the cell-of-origin (intestinal epithelial cell, left arrow) and tumor or non-cell autonomous effects by preventing immune cell activation and inflammation, which can occur directly (middle arrow) or by maintaining the barrier function of the epithelium (right arrow). 

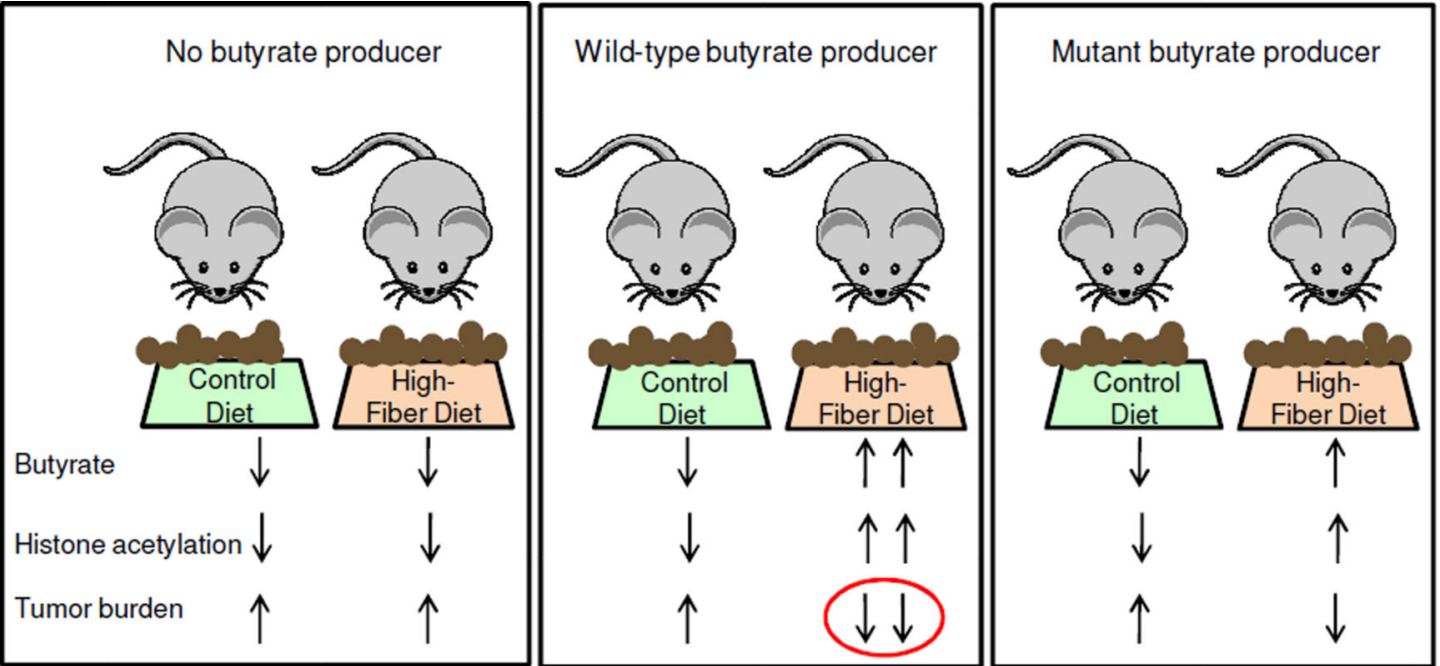
No butyrate producer

Figure 5.

Dietary fiber protects against colorectal cancer in a microbiota- and butyrate-dependent manner in a gnotobiotic mouse model. Mice were colonized with several bacteria that either excluded or included a butyrate producer, Butyrivibrio fibrisolvens, as indicated at the top. A wild-type and mutant strain of B. fibrisolvens utilized in separate gnotobiotic isolators (depicted by boxes around each group of mice). In each isolator, the mice received control or high-fiber diets ( $6 \%$ fructo-oligosaccharides/inulin but otherwise identical to the control diet) except for a butyrate-fortified diet in the isolator at the far right. Arrows at the bottom indicate relative levels of luminal butyrate along with global histone acetylation levels and tumor burden following AOM treatment. Butyrate production was attenuated, but not completely abolished, in the mutant strain when provided a high-fiber diet as denoted by one upward butyrate arrow instead of a downward arrow or two upward arrows. The ovals highlight experimental conditions that yield a lower tumor burden, and this correlates with higher butyrate levels and histone acetylation levels. 


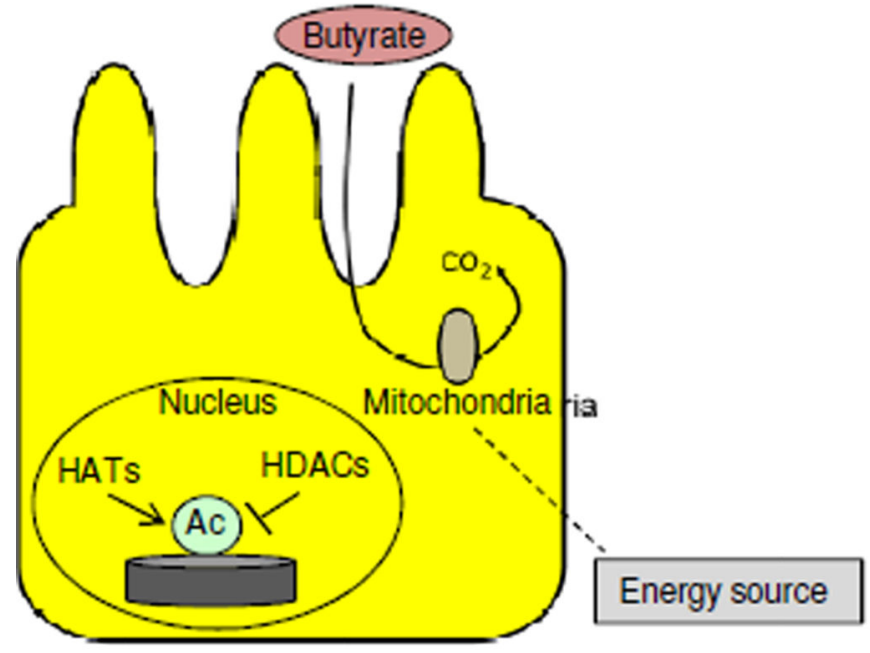

Normal colonocyte

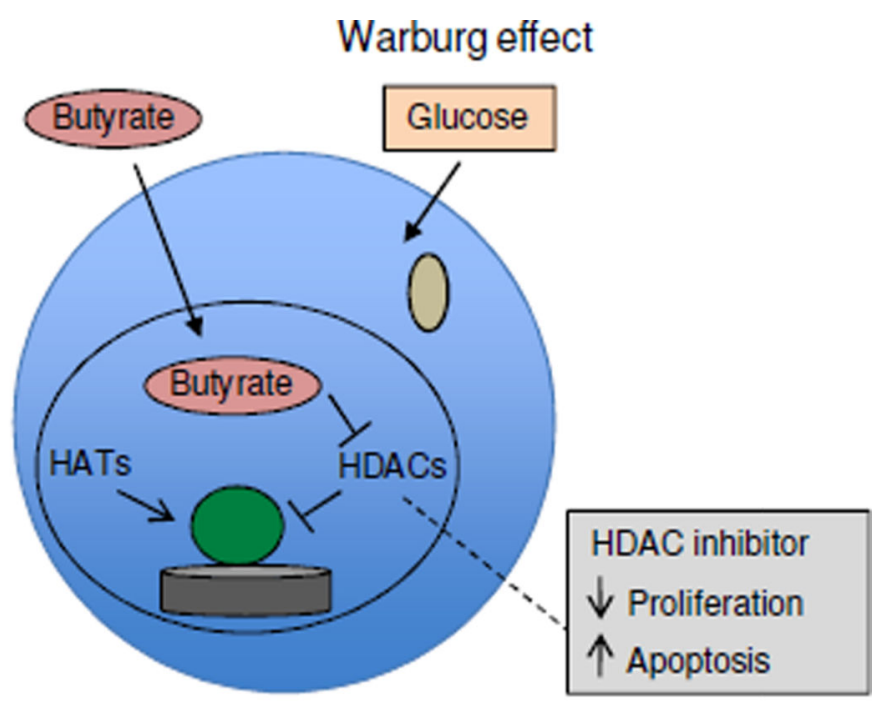

Cancerous colonocyte

Figure 6.

Mechanism of butyrate-mediated tumor suppression. In normal colonocytes (left), butyrate is utilized as the primary energy source and metabolized in the mitochondria so relatively little accumulates inside of the cell. In cancerous colonocytes (right), glucose is the primary energy source due to the Warburg effect. Butyrate is still transported into the cell via monocarboxylate transporters but is not metabolized in the mitochondria, which allows it to accumulate in the nucleus and function as an HDAC inhibitor to epigenetically regulate genes involved in cell proliferation and apoptosis. 


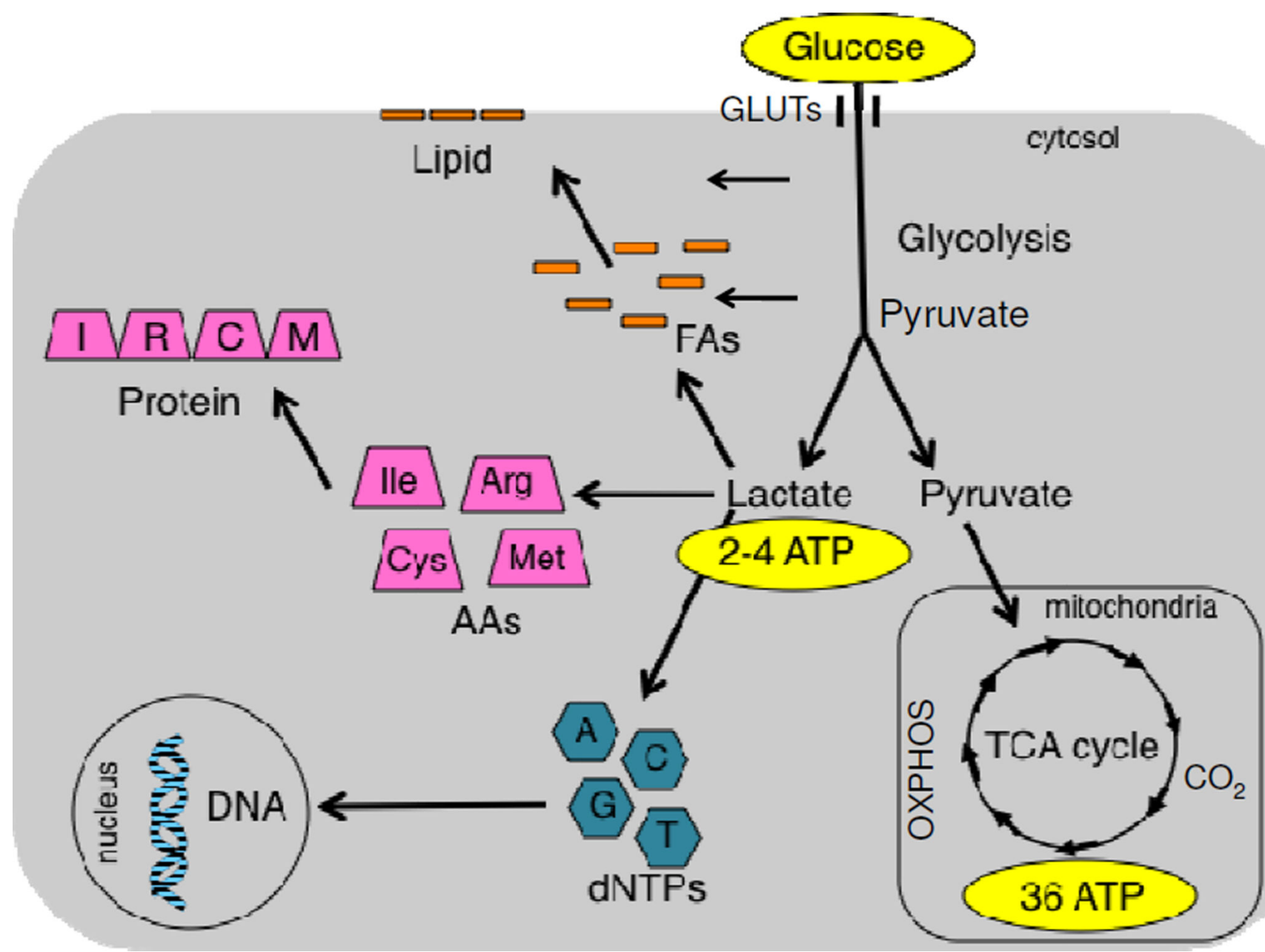

Figure 7.

Cancer cells undergo the Warburg effect to support their rapid proliferation. Normal cells that are not transformed usually metabolize glucose by undergoing oxidative metabolism in the mitochondria (TCA cycle and OXPHOS), which yields 36 ATPs per glucose molecule. In contrast, cancer cells undergo aerobic glycolysis and generate lactate as an end product, which yields 4 ATPs per glucose molecule (only 2 ATPs if oxygen is limited in a hypoxic environment). The cancer cell makes up for this inefficient energy production mechanism by upregulating glucose transporters (GLUTs) and increasing glucose uptake, and this is the basis for tumor imaging in the clinic (via FDG-PET). Because glucose is not metabolized completely to $\mathrm{CO}_{2}$, the pentose phosphate pathway and salvage pathways scavenge carbons and nitrogens from glycolytic intermediates to replenish the pools of dNTPs, amino acids (AAs), and fatty acids (FAs). This serves a conduit for biosynthetic pathways to replicate DNA in the nucleus, all of the proteins in the proteome, and lipids. The rationale behind this strategy is that acquiring the raw materials to double cellular biomass is a more important challenge for rapidly dividing cancer cells than generating sufficient ATP levels. 


\section{Chemoprevention (Probiotics/prebiotics)}

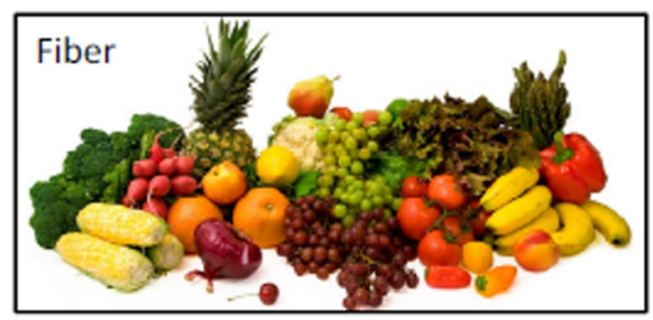

Endogenous HDAC inhibitor (butyrate):

\section{Chemotherapy}

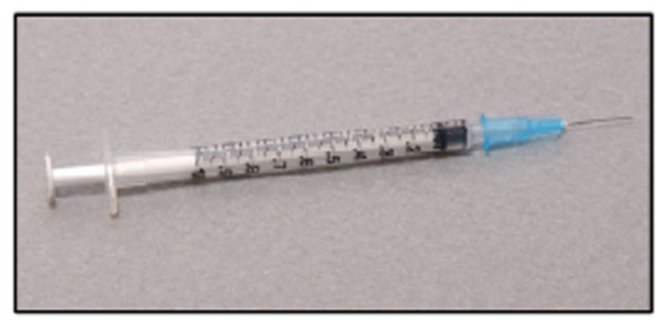

Synthetic HDAC inhibitors (e.g., vorinostat/SAHA, romidepsin):

1. Bioavailability restricted to colon

\section{Delivered systemically}

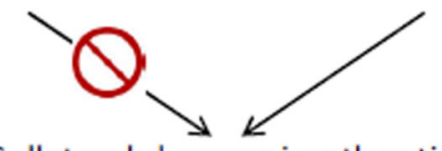

Collateral damage in other tissues

\section{Naturally occurring fatty acid}

\section{Synthetic molecules}

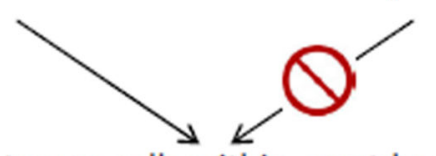

Targets tumor cells within crypt because of Warburg effect

\section{Figure 8.}

Chemoprevention versus chemotherapy. Probiotics/prebiotics can increase levels of butyrate, which is an endogenous HDAC inhibitor that does not have adverse effects associated with synthetic HDAC inhibitors used in chemotherapy because its bioavailability is primarily restricted to the colon and it targets tumor cells because of the Warburg effect. See text for further explanation. 
Table 1

Bacterial metabolites derived from food components reported to have chemopreventive effects

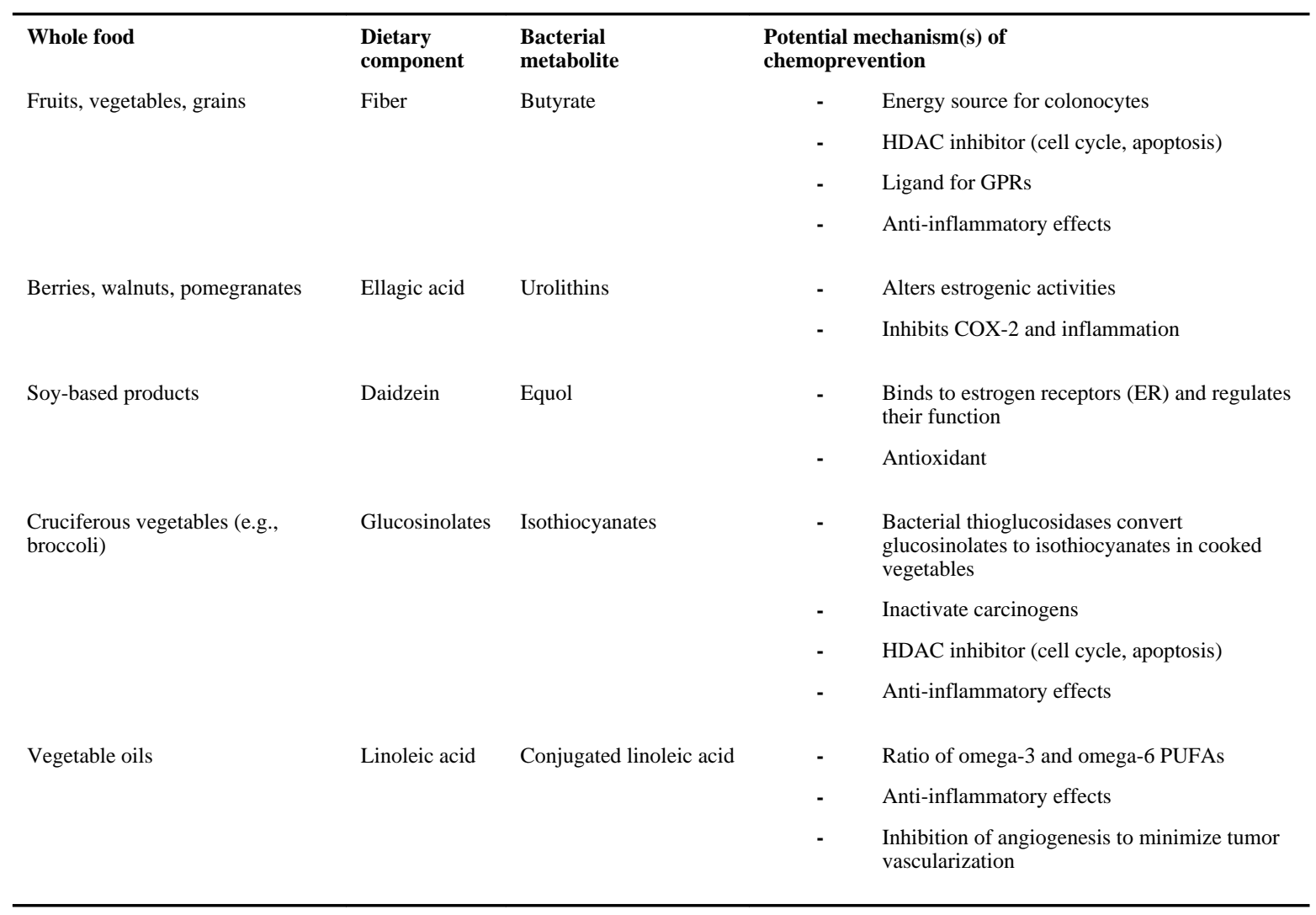

